Cite this: Phys. Chem. Chem. Phys., 2013,

\title{
Irreversible thermochromism in copper chloride Imidazolium Nanoparticle Networks $†$
}

15, 12717

\author{
Martin Kronstein, ${ }^{a}$ Konstantin Kriechbaum, ${ }^{a}$ Johanna Akbarzadeh, ${ }^{b}$ \\ Herwig Peterlik ${ }^{b}$ and Marie-Alexandra Neouze ${ }^{* a c}$
}

Received 30th January 2013, Accepted 22nd May 2013

DOI: $10.1039 /$ c3cp50430a

www.rsc.org/pccp

\begin{abstract}
In this work Imidazolium Nanoparticle Networks (INNs) with chloride counter-ions were used to complex copper dichloride. This complexation reaction leads to the formation of a green material. The properties of the copper INN material were compared to: first, copper imidazolium complexes, without the presence of silica nanoparticles, which are not thermochromic; second, chloride-containing INN material. The copper INN material showed irreversible thermochromic behaviour, with a clear colour change from green to yellow at $180{ }^{\circ} \mathrm{C}$, which is due to a configuration change of the copper complex from planar to tetragonal. This structural change was studied using DSC and in situ SAXS measurements during heat treatment. The thermochromic material is stable under air up to $250{ }^{\circ} \mathrm{C}$. This preliminary study opens the door of optical sensors for INN materials.
\end{abstract}

\section{Introduction}

In recent years material chemists have dedicated great efforts to develop nanoparticle networks. ${ }^{1-4}$ Nanoparticle networks are materials mainly consisting of nanoparticles, mainly inorganic, whose assembly or self-assembly is strictly controlled in order to hinder aggregation of the nanoparticles. This control can be achieved using various methods, such as template assisted assembly $^{5,6}$ or assembly driven by molecular linkers. ${ }^{7-9}$ These materials have already proven to be highly interesting for applications as varied as plasmonics, ${ }^{10,11}$ magnetic materials, ${ }^{12}$ water treatment ${ }^{13}$ or catalysis. ${ }^{14}$ In addition the fundamental understanding of the assembly driving forces and interactions of nanoparticles is intrinsically interesting.

In this context we reported a new material, referred to as the Imidazolium Nanoparticle Network (INN), consisting of silica nanoparticles linked by imidazolium units. ${ }^{15-17}$ The INN material showed special features, such as catalytic activity or anion

\footnotetext{
${ }^{a}$ Vienna University of Technology, Institute of Materials Chemistry, 1060 Vienna, Austria

${ }^{b}$ University of Vienna, Faculty of Physics, 1090 Vienna, Austria

${ }^{c}$ Interdisciplinary Laboratory on nanometric and supramolecular organization (LIONS), CEA Saclay, DSM, IRAMIS, SIS2M, 91191 Gif-sur-Yvette Cedex, France. E-mail: marie-alexandra.gauthey-neouze@cea.fr; Fax: +43158801 165988; Tel: +43158801 165206

$\dagger$ Electronic supplementary information (ESI) available: DLS of the $\mathrm{SiO}_{2}$ nanoparticles. Powder XRD spectrum of $\mathbf{C u C l}_{\mathbf{4} \_} \mathbf{I N N}$. Heating system of the dip-coated film to observe the color change. Luminescence spectra of $\mathbf{C l}_{-} \mathbf{I N N}$ and

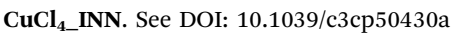

exchange ability, owing to the imidazolium organic linker, which can be considered as an ionic liquid-like linker. The use of INN for catalysis is also strongly facilitated owing to the presence of the nanoparticles, leading to a solid-state catalyst; which allows an easy filtering of the solid-state catalysts after the reaction. ${ }^{18}$ In addition it turned out that the presence of the nanoparticles can drive, even force, the organization of the imidazolium linkers within the material. ${ }^{19,20}$ The organization of the inter-particle imidazolium ligands occurs through $\pi-\pi$ stacking of the imidazolium units (Scheme 1). This forced-organization led to new properties such as intense luminescence of the INN materials. $^{19}$ The amount of luminescence was proven to depend on the degree of the short-range order (SRO) of the ligands within the INN material. The SRO can easily be determined using small angle X-ray scattering, which thus helps to relate structural and functional properties of weakly ordered or non-crystalline nanostructure materials. ${ }^{19-23}$

Nanoparticle networks are also considered for sensoric applications. $^{24,25}$ Besides, some complexes such as nickel(II) or copper(II) complexes are promising for application in sensors owing to their possible thermochromic behavior. ${ }^{26-28}$ For example, diethylammonium chloride could easily complex copper dichloride salts to form thermochromic diethylammonium copper tetrachloride species. At room temperature the copper center in the diethylammonium copper tetrachloride complex adopts a square planar configuration with the help of coordinating solvent molecules. The square planar copper tetrachloride complexes form green crystals. When the temperature increases, the copper tetrachloride switches to a tetrahedral configuration with a lower band 


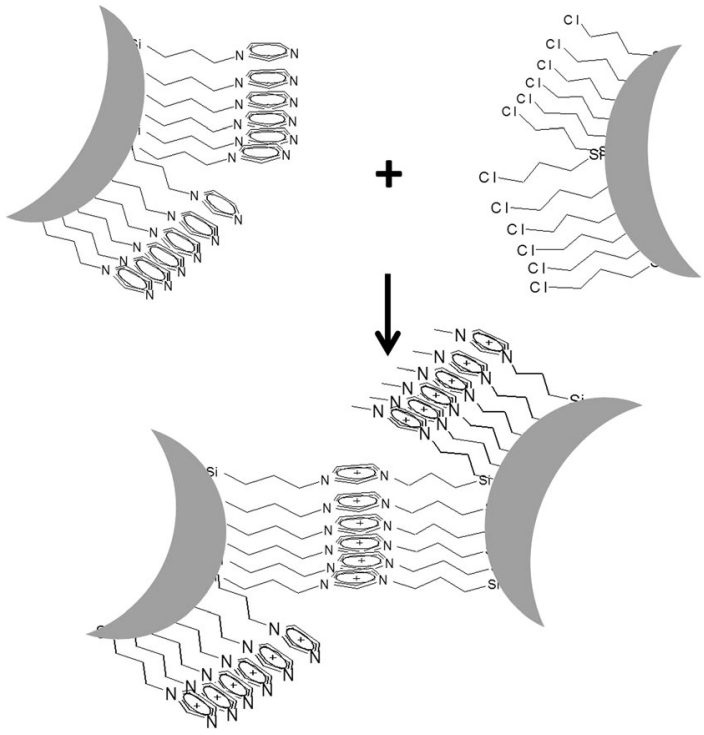

Scheme 1 Reaction of imidazole modified silica nanoparticles with chloroalkyl modified silica nanoparticles to form Imidazolium Nanoparticle Networks (INN).

gap as the interaction with the solvent molecules is weakening. As a consequence a transition is observed at $52{ }^{\circ} \mathrm{C}$ for the diethylammonium copper tetrachloride where the crystals are changing color from green to yellow. Thermochromism was observed with metal ammonium complexes as discussed above, ${ }^{26}$ but very few thermochromic metal imidazolium compounds were reported. ${ }^{27,29,30}$ One can cite the investigation performed by Bhattacharya et al. on the role of water in the thermochromism of benzimidazolium tetrachlorocuprates. ${ }^{31}$ This benzimidazolium tetrachlorocuprate shows thermochromic behavior, however its stability is limited to $140{ }^{\circ} \mathrm{C}$.

Literature reporting ionic liquid like ligands, such as imidazolium or pyridinium, immobilized on silica and used as catalysts after complexation of metal centers like copper, did not show thermochromic behavior. ${ }^{17,18,32,33}$

In this article we present the complexation of copper dichloride salt by Imidazolium Nanoparticle Networks to form thermochromic copper(II) tetrachloride Imidazolium Nanoparticle Networks. The structural and thermochromic properties of the material are investigated and compared to two reference materials: the first reference compound was obtained from the reaction of copper dichloride with the equivalent 1,3-butylmethylimidazolium chloride without nanoparticles. And the second reference material is the INN before reaction with copper dichloride (CI_INN).

\section{Experimental}

\section{Chemicals}

All starting chemicals were of reagent grade and used as purchased.

\section{Syntheses}

Synthesis of the silica nanoparticles $\left(\mathrm{SiO}_{2}\right)$. In a $250 \mathrm{~mL}$ round bottom flask, $60 \mu \mathrm{L}(0.01 \mathrm{~mol})$ of ammonia solution
$(32 \%)$ and $1.98 \mathrm{~g}(0.11 \mathrm{~mol})$ of water are added to $100 \mathrm{~mL}$ of absolute methanol. $10.41 \mathrm{~g}(0.05 \mathrm{~mol})$ of TEOS are added dropwise under stirring. The final solution is stirred for three days at room temperature. The resulting silica nanoparticles have an average diameter of $16 \mathrm{~nm}$.

Synthesis of 3-iodopropyltrimethoxysilane. The synthesis is carried out under an argon atmosphere. Sodium iodide $(45.0 \mathrm{~g}$, $0.3 \mathrm{~mol}$ ) is dissolved in $150 \mathrm{~mL}$ of absolute acetone. 3-Chloropropyltrimethoxysilane $(48.9 \mathrm{~g}, 0.246 \mathrm{~mol})$ is added dropwise under stirring. The mixture is refluxed under stirring overnight. The precipitated sodium chloride is filtered off under an argon atmosphere. Then the product is distilled at $52{ }^{\circ} \mathrm{C}$ under vacuum conditions ( $3 \mathrm{mbar}$ ). The resulting 3 -iodotrimethoxysilane is a yellowish liquid.

${ }^{1} \mathrm{H}$ NMR (250 MHz, $\left.\mathrm{CDCl}_{3}\right): \delta(\mathrm{ppm}) 3.58\left(\mathrm{~s}, 9 \mathrm{H}, \mathrm{Si}\left(\mathrm{OCH}_{3}\right)_{3}\right)$; $3.22\left(\mathrm{t}, 2 \mathrm{H}, \mathrm{I}-\mathrm{CH}_{2}-\right)$; 1.93 (q, 2H, I- $\left.\mathrm{CH}_{2}-\mathrm{CH}_{2}-\mathrm{CH}_{2}-\right) ; 0.75$ (t, $2 \mathrm{H}$, $-\mathrm{CH}_{2}-\mathrm{Si}$.

Synthesis of $\boldsymbol{N}$-(3-propyltrimethoxysilane)imidazole. The synthesis is carried out under an argon atmosphere. Sodium hydride $(2.9 \mathrm{~g}, 0.12 \mathrm{~mol})$ is dissolved in $150 \mathrm{~mL}$ of absolute THF, the mixture is cooled to approximately $0{ }^{\circ} \mathrm{C}$ using an ice bath. Imidazole $(8.3 \mathrm{~g}, 0.12 \mathrm{~mol})$ is added slowly under stirring. After complete addition of the imidazole, the ice bath is removed and the mixture is maintained under stirring until no more hydrogen gas is produced. Then $23.12 \mathrm{~g}(0.09 \mathrm{~mol})$ of 3 -iodopropyltrimethoxysilane are added and the mixture is maintained at reflux overnight. The orange suspension is filtered off under an argon atmosphere and the solvent is removed under vacuum conditions. By addition of $150 \mathrm{~mL}$ of absolute dichloromethane a colorless precipitate appears and is filtered off under an argon atmosphere. Finally, the product is distilled at $105{ }^{\circ} \mathrm{C}$ under vacuum (3 mbar). $\mathrm{N}$-(3-Propyltrimethoxysilane)imidazole is obtained as a colorless and transparent liquid.

${ }^{1} \mathrm{H}$ NMR (250 MHz, $\left.\mathrm{CDCl}_{3}\right): \delta$ (ppm) $7.54(\mathrm{~s}, 1 \mathrm{H},-\mathrm{N}-\mathrm{CH}-\mathrm{N}-)$; $7.01(\mathrm{~s}, 1 \mathrm{H},-\mathrm{N}-\mathrm{CH}-\mathrm{CH}-\mathrm{N}=) ; 6.88(\mathrm{~s}, 1 \mathrm{H},-\mathrm{N}-\mathrm{CH}-\mathrm{CH}-\mathrm{N}=)$; $3.88\left(\mathrm{t}, 2 \mathrm{H},=\mathrm{N}-\mathrm{CH}_{2}-\mathrm{CH}_{2}-\right) ; 3.53\left(\mathrm{~s}, 9 \mathrm{H}, \mathrm{Si}\left(\mathrm{OCH}_{3}\right)_{3}\right) ; 1.83(\mathrm{q}, 2 \mathrm{H}$, $\left.=\mathrm{N}-\mathrm{CH}_{2}-\mathrm{CH}_{2}-\mathrm{CH}_{2}-\mathrm{Si}\right) ; 0.54\left(\mathrm{t}, 2 \mathrm{H},-\mathrm{CH}_{2}-\mathrm{Si}\right)$.

${ }^{13} \mathrm{C}$ NMR (250 MHz, $\left.\mathrm{CDCl}_{3}\right): \delta$ (ppm) $136.8(-\mathrm{N}-\mathrm{CH}-\mathrm{N}-)$; 128.1 $(-\mathrm{N}-\mathrm{CH}-\mathrm{CH}-\mathrm{N}=) ; 120.7(-\mathrm{N}-\mathrm{CH}-\mathrm{CH}-\mathrm{N}=) ; 56.2\left(-\mathrm{Si}\left(\mathrm{OCH}_{3}\right)_{3}\right) ; 55.7$ $\left(=\mathrm{N}-\mathrm{CH}_{2}-\mathrm{CH}_{2}-\right) ; 25.1$ ( $\left.=\mathrm{N}-\mathrm{CH}_{2}-\mathrm{CH}_{2}-\mathrm{CH}_{2}-\mathrm{Si}\right) ; 7.4\left(-\mathrm{CH}_{2}-\mathrm{Si}\right)$.

Synthesis of chloropropyl modified silica nanoparticles (SiO__Cl). $32 \mathrm{~mL}$ of the previously prepared silica nanoparticle solution are degassed under vacuum for several minutes to remove excessive ammonia. Then $2.84 \mathrm{~g}$ (14.29 mmol) of 3-chloropropyltrimethoxysilane are added dropwise. The solution is stirred at room temperature for 24 hours.

Synthesis of $\boldsymbol{N}$-propylimidazole modified silica nanoparticles ( $\mathrm{SiO}_{2}$ Im). $32 \mathrm{~mL}$ of the previously prepared silica nanoparticle solution are degassed under vacuum for several minutes to remove excessive ammonia. Then $3.3 \mathrm{~g}(14.29 \mathrm{mmol})$ of $N$-(3-propyltrimethoxysilane)imidazole are added dropwise. The solution is stirred at room temperature for 24 hours.

Networking of the silica nanoparticles, formation of the imidazolium chloride bridging unit (Cl_INN). $16 \mathrm{~mL}$ solution of silica nanoparticles modified with $\mathrm{N}$-(3-propyltrimethoxysilane)imidazole and $16 \mathrm{~mL}$ solution of silica nanoparticles modified 
with 3-chloropropyltrimethoxysilane are transferred to a $100 \mathrm{~mL}$ round bottom flask. Then $10 \mathrm{~mL}$ of dry methanol are added. The solution is stirred for 2 days at room temperature and is finally dried under vacuum conditions ( 3 mbar). A transparent gel is obtained.

Synthesis of $\mathrm{CuCl}_{4}$ INN. $0.15 \mathrm{~g}(0.85 \mathrm{mmol})$ of $\mathrm{CuCl}_{2} \cdot 2 \mathrm{H}_{2} \mathrm{O}$ were added to $17 \mathrm{~mL}$ of the Cl_INN solution in a $100 \mathrm{~mL}$ round bottom flask. The solution was stirred overnight at room temperature and was finally dried under vacuum. A green transparent gel was obtained.

Synthesis of bis-(butylmethylimidazolium) copper tetrachloride ((BuMeIm) $\left.)_{2} \cdot \mathbf{C u C l}_{4}\right) .3 \mathrm{~g}(36.5 \mathrm{mmol})$ of 1-methylimidazole were mixed with $3.38 \mathrm{~g}(36.5 \mathrm{mmol})$ of 1-chlorobutane and $5 \mathrm{~mL}$ of ethylacetate. $3.11 \mathrm{~g}(18.25 \mathrm{mmol})$ of $\mathrm{CuCl}_{2} \cdot 2 \mathrm{H}_{2} \mathrm{O}$ were added to the previously synthesized butylmethylimidazolium chloride ( $36.5 \mathrm{mmol})$. By adding $\mathrm{CuCl}_{2} \cdot 2 \mathrm{H}_{2} \mathrm{O}$ the temperature rose highly and the color changed to dark blue. After a few minutes the solution was cooled and the color began to turn green. The solution was stirred at room temperature over night and the resulting water was visible as a second, transparent phase. The product was washed with water and dried under vacuum conditions.

\section{Characterizations}

Nuclear magnetic resonance (NMR). Solution NMR spectra were recorded on a Bruker Avance $300\left({ }^{1} \mathrm{H}\right.$ at $300.13 \mathrm{MHz}$ and ${ }^{13} \mathrm{C}$ at $75.40 \mathrm{MHz}$ ) equipped with a $5 \mathrm{~mm}$ inverse-broadband probe head with a $z$-gradient unit.

Electron dispersive X-ray (EDX) measurements. The sample was covered before measurement by a carbon sputtering. SEM measurements were performed on a JEOL 5410 connected to an EDX detector RÖNTEC.

Thermogravimetric analysis (TGA). The analyses were carried out using a NETZSCH TG 209 at a heating rate of $10{ }^{\circ} \mathrm{C} \mathrm{min}-1$ under air.

Differential scanning calorimetry (DSC). For the analysis, on a Mettler Toledo DSC 823e, a few milligrams of sample were sealed in an aluminum crucible under a nitrogen atmosphere. The temperature program was recorded at a speed of $5{ }^{\circ} \mathrm{C} \mathrm{min}{ }^{-1}$ and consisted of a first heating phase from room temperature to $250{ }^{\circ} \mathrm{C}$, followed by a cooling phase from $250{ }^{\circ} \mathrm{C}$ to room temperature.

X-ray powder diffraction (XRD). X-ray powder diffraction (XRD) measurements were performed on a Philipps X'Pert diffractometer using the $\mathrm{Cu}-\mathrm{K} \alpha$ radiation $(\lambda=1.542 \AA)$.

Small-angle X-ray scattering (SAXS). Small-angle X-ray scattering (SAXS) was performed using a rotating anode generator equipped with a pinhole camera (Nanostar from Bruker AXS, Karlsruhe, with $\mathrm{Cu} \mathrm{K} \alpha$ radiation from crossed Göbel mirrors). The X-ray patterns were recorded using an area detector (VANTEC 2000) and radially averaged to obtain the scattering intensity depending on the scattering vector $\boldsymbol{q}=(4 \pi / \lambda) \sin \theta$, with $2 \theta$ being the scattering angle and $\lambda=0.1542 \mathrm{~nm}$ the X-ray wavelength.

For the in situ experiments, the samples were transferred into quartz glass capillaries with $1.5 \mathrm{~mm}$ outer diameter and 10 micron wall thickness (from Hilgenberg, Germany) and placed into an X-ray transparent furnace. The samples were then heated at a heating rate of less than $2{ }^{\circ} \mathrm{C}$ per minute and an accuracy of $0.5{ }^{\circ} \mathrm{C}$, stepwise, and at each temperature step $\left(5{ }^{\circ} \mathrm{C}\right.$ intervals) a pattern was taken for $900 \mathrm{~s}$.

Dynamic light scattering (DLS). For the measurement, the solid was suspended in ethanol. The DLS experiments were carried out without prior sonication of the samples. The run time of the measurements is 10 seconds. Every size distribution curve is obtained by averaging 10 measurements. The apparatus is an ALV/CGS-3 compact goniometer system, equipped with an ALV/LSE-5003 light scattering electronics and a multiple $\tau$ digital correlator, and a $632.8 \mathrm{~nm}$ JDSU laser 1145P.

Dip-coating. The thin film syntheses were made by dip-coating on glass substrates (withdrawal rate $24 \mathrm{~cm} \mathrm{~min}^{-1}$ ). Before coating the microscope slab was washed with acetone, ethanol and water.

Kofler bench. The color change experiments using a microscope were carried out on a heating plate.

Digital photos. Digital photos were made using a Nikon D5000. The digital photos on the heating plate were made using a Canon ixus 70.

\section{Results and discussion}

The starting Imidazolium Nanoparticle Network with a chloride counter-anion (Cl_INN) was prepared using a reported method. ${ }^{16,34}$ First, silica nanoparticles with an average diameter of $16 \mathrm{~nm}$ were prepared using an ammonia catalyzed sol-gel process in ethanol (see ESI $\dagger$ ). After elimination of the ammonia by stirring, the suspension of silica nanoparticles was divided into two vessels. The silica nanoparticles were modified, in the first batch by (chloropropyl)trimethoxysilane, and by an equimolar amount of $\mathrm{N}$-(3-trimethoxysilylpropyl)imidazole in the second batch. The two batches were then mixed together and the functional groups, chloro-alkyl and imidazole, reacted under stirring at room temperature overnight (Scheme 1).

Copper dichloride dihydrate was added to the methanolic suspension of Cl_INN (Fig. 1, top). The amount of copper

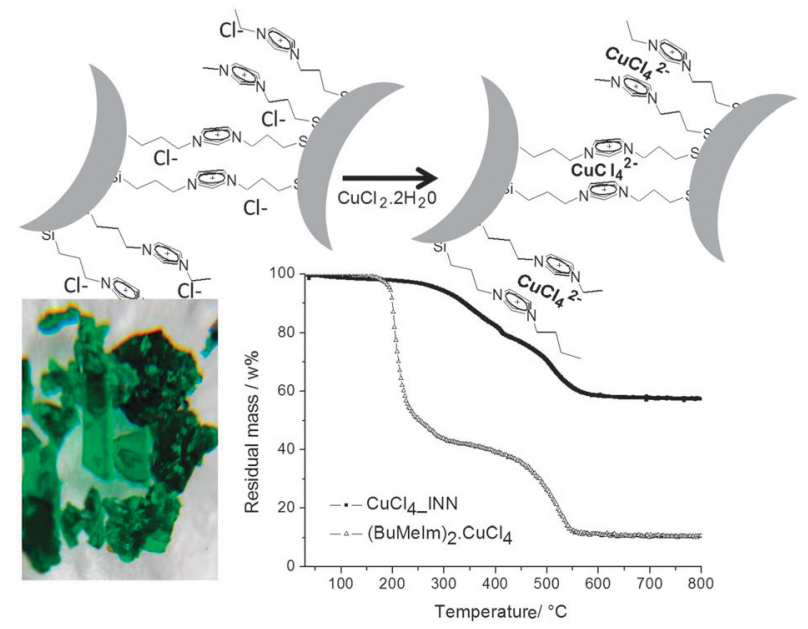

Fig. 1 (top) Formation of $\mathrm{CuCl}_{4}$ INN after complexation of cupper dichloride dihydrate by $\mathrm{Cl}_{-} \mathbf{I N N}$. (bottom left) Green $\mathbf{C u C l}_{4}$ INN. (bottom right) Thermogravimetric behavior of $\mathrm{CuCl}_{4} \mathrm{INN}$ and $(\mathrm{BuMeIm})_{2} \cdot \mathrm{CuCl}_{4}$. 
dichloride added to the suspension, in order to get a 1:2 molar ratio of copper: imidazolium, corresponded to half the molar amount of $\mathrm{N}$-(3-trimethoxysilylpropyl)imidazole introduced for the preparation of the Cl_INN. We have shown in a previous work that up to $82 \%$ of the chlorine atoms contained in the Cl_INN material could be exchanged by tetrafluoroborate anions. As only chloride as an anionic imidazolium counter anion can be exchanged, and not the covalently linked chlorine in the precursor (chloropropyl)trimethoxysilane, this indicates that $82 \mathrm{~mol} \%$ or more of the $N$-(3-trimethoxysilylpropyl)imidazole and (chloropropyl)trimethoxysilane have reacted to form the imidazolium linkers in Cl_INN. The suspension was stirred overnight at room temperature. After washing and drying a green transparent gel was obtained (Fig. 1, bottom left). The product will be referred hereafter to as $\mathbf{C u C l}_{\mathbf{4}} \mathbf{I N N}$.

The thermal stability of the new green material was determined by thermo gravimetric analysis. No degradation could be observed before $250{ }^{\circ} \mathrm{C}$ under air (Fig. 1, bottom right). Furthermore, no crystalline copper salt, either di- or tetra-chloride, could be observed in the powder XRD spectrum, only the very broad reflection characteristic of amorphous silica could be observed around $20^{\circ}$ (see ESI $\dagger$ ).

EDX measurement delivered a rough idea of the atomic composition of the material, indicating a 1:4:35 molar ratio between $\mathrm{Cu}: \mathrm{Cl}$ : C. These numbers should be taken with caution, as the error in determining molar ratios using EDX for materials ranging from more heavy atoms $(\mathrm{Cu})$ to very light atoms $(\mathrm{C}, \mathrm{Cl})$ is not negligible. However, the ratio of $\mathrm{Cu}$ to $\mathrm{Cl}$ is close to the value of $1: 4$, which was expected for copper tetrachloride. The high amount of carbon atoms is attributed to three reasons: first, the error in the method is higher for lighter atoms; second, the carbon atoms from the ligand chains which did not react into imidazolium groups did not coordinate copper atoms; and third, some additional carbon atoms belong to side groups from the slightly incomplete condensation of the alkoxysilanes.

Similar to Cl_INN before complexation, the $\mathbf{C u C l}_{\mathbf{4} \_\mathbf{I N N}}$ material can be processed into thin films (Fig. 2). This was realized by slowly evaporating the green suspension to reduce the volume of solvent. Finally, the concentrated suspension was dip-coated onto glass slabs, previously activated with concentrated basic solution and washed with water, ethanol and then acetone.

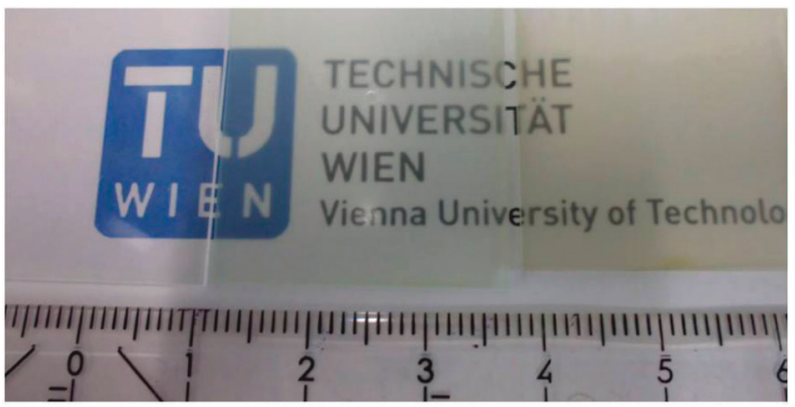

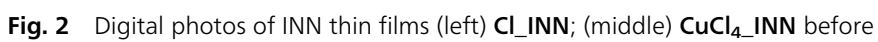
heating; (right) $\mathrm{CuCl}_{4}$ INN after heating at $180^{\circ} \mathrm{C}$.
Thermochromic behavior of $\mathbf{C u C l}_{\mathbf{4}}$ INN was investigated by depositing a dip-coated glass slab on a heating plate and increasing the temperature (see ESI $\dagger$ ). A clear color change could be observed when the temperature reached $190{ }^{\circ} \mathrm{C}$ : the film remained transparent but turned from slightly green (Fig. 2, middle) to yellow (Fig. 2, right).

Optical microscopy with a Kofler bench revealed a color

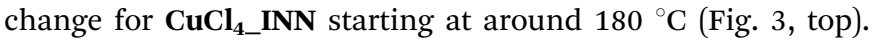
However, after returning to room temperature, the crystals kept the yellow color: thus, the thermochromic behavior is not reversible. To identify the structural origin, i.e. a possible phase transformation, we used differential scanning calorimetry (DSC). The DSC curve during heating at a ramp rate of $5{ }^{\circ} \mathrm{C}$ per minute from $40{ }^{\circ} \mathrm{C}$ to $250{ }^{\circ} \mathrm{C}$ showed a strong endothermic peak centered at $180{ }^{\circ} \mathrm{C}$ (Fig. 3, bottom). It is very probable that this corresponds to the change in configuration of the copper(II) tetrachloride from a square planar configuration to a compressed tetrahedral configuration, as known for thermochromic copper(II) chloride compounds. ${ }^{35}$ During cooling at the identical ramp rate as in the heating cycle, the curve remains flat and no change can be observed. Further heating-cooling cycles showed no further color change, suggesting no further modification of the copper complex in the material.

For comparison the equivalent compound without nanoparticles was prepared, the bis(butylmethylimidazolium)copper(II) tetrachloride referred to as $(\mathbf{B u M e I m})_{2} \cdot \mathbf{C u C l}_{4}$. The procedure was similar to the one performed for the $\mathbf{C u C l}_{\mathbf{4} \_} \mathbf{I N N}$ material (Fig. 4). Equimolar amounts of 1-methylimidazole and 1-chlorobutane were reacted in ethylacetate at room temperature overnight. After evaporating the solvent, copper(II) dichloride dihydrate was added in a 1:2 molar ratio between the copper salt and the butylmethylimidazolium chloride. Immediately after mixing, the temperature increased considerably, indicating an exothermic chemical reaction. At the same time, the color changed from blue, due to unreacted copper dichloride salt, to deep green. The mixture was stirred overnight at room temperature. As the reaction was carried out without solvent, the elimination of water, from the copper(II)

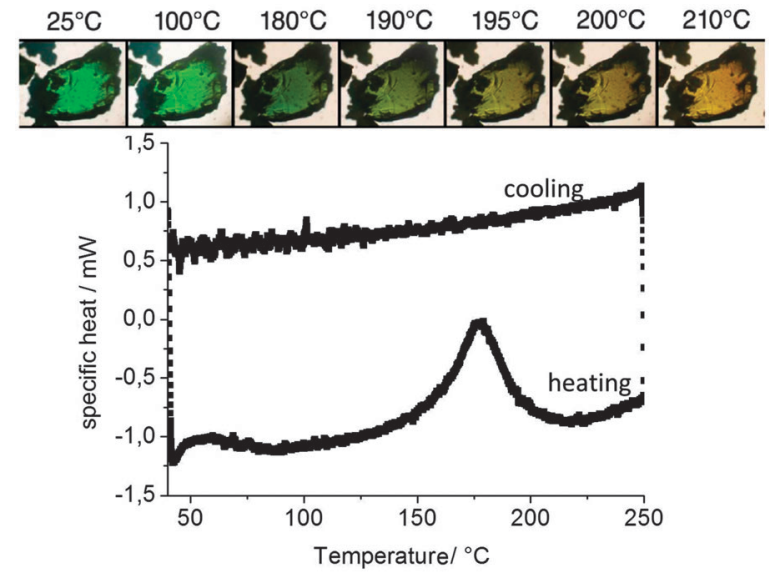

Fig. 3 (top) Microscope observation of the color change of $\mathbf{C u C l}_{4}$ INN on a Kofler bench and (bottom) the DSC curve for $\mathrm{CuCl}_{4}$ INN under heating and cooling. 


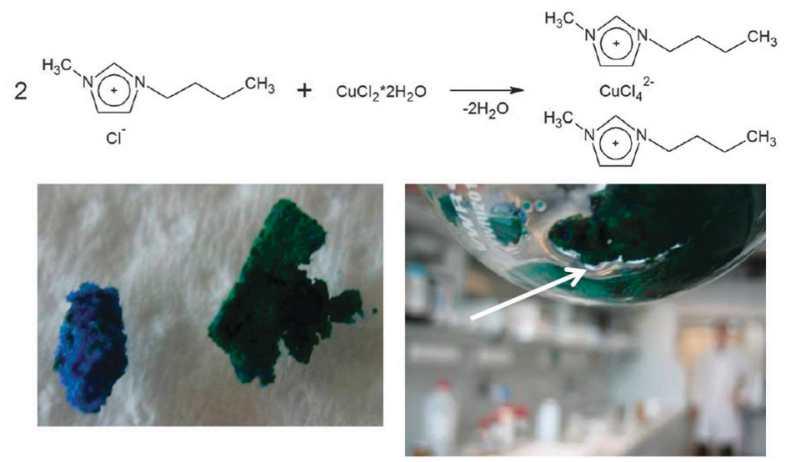

Fig. 4 (top) Reaction pathway for the preparation of (BuMelm) $2 \cdot \mathrm{CuCl}_{4}$. (bottom left) Photo of the imidazolium chloride and copper dichloride dihydrate mixture (left) before and (right) after complexation; (bottom right) photo of the water elimination characteristic of the complexation reaction.

dichloride dihydrate, could be easily observed in the range of the compound (Fig. 4, inset).

The thermal behavior of this reference compound $(\mathbf{B u M e I m})_{2} \cdot \mathbf{C u C l}_{\mathbf{4}}$ was investigated under the same conditions as the $\mathbf{C u C l}_{\mathbf{4}} \mathbf{I N N}$ material. TGA analysis showed distinct degradation steps (Fig. 1, bottom right). A first step occurred in the temperature range from $166{ }^{\circ} \mathrm{C}$ to $323{ }^{\circ} \mathrm{C}$, and a second step occurred from $350{ }^{\circ} \mathrm{C}$ up to $550{ }^{\circ} \mathrm{C}$. Only $11 \mathrm{wt} \%$ were remaining after degradation, which corresponds to the copper(II) oxide residues. Comparing (BuMeIm) $)_{2} \cdot \mathbf{C u C l}_{4}$ and $\mathbf{C u C l}_{4} \_$INN, a clear gain in stability was observed, arising from the formation of the nanoparticle network, as already observed for other INN materials (Cl_INN). ${ }^{15,16}$ The shift of the onset temperature of decomposition between (BuMeIm) $)_{2} \cdot \mathbf{C u C l}_{4}$ and $\mathbf{C u C l}_{4} \mathbf{I N N}$ is larger than $100{ }^{\circ} \mathrm{C}$.

By heating the green powder (BuMeIm) $)_{2} \cdot \mathbf{C u C l}_{4}$ on a Kofler bench under an optical microscope, melting was observed at around $140{ }^{\circ} \mathrm{C}$, corresponding to the first endothermic peak in DSC (Fig. 5). After the melting, towards higher temperatures the color of the compound turned to brown. This observation is in good agreement with the decomposition temperature of $166{ }^{\circ} \mathrm{C}$

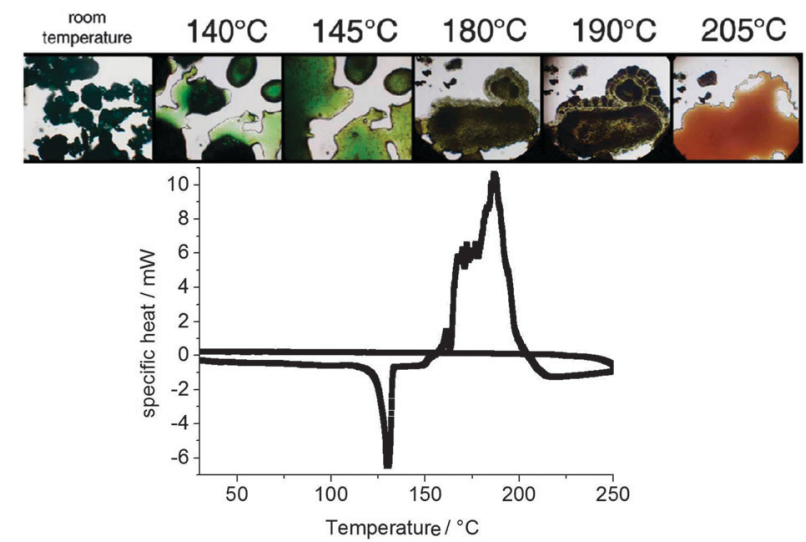

Fig. 5 (top) Microscope observation of the thermal behavior of (BuMelm) 2 $\mathrm{CuCl}_{4}$ on a Kofler bench and (bottom) the DSC curve for (BuMelm) ${ }_{2} \cdot \mathrm{CuCl}_{4}$ under heating and cooling. observed in the TGA analysis (Fig. 1, bottom right) cooling the sample to room temperature showed that the process is irreversible.

The structural development at the nanometer scale of the two reference compounds, Cl_INN and (BuMeIm) $)_{2} \cdot \mathbf{C u C l}_{4}$, and of the thermochromic material, $\mathbf{C u C l}_{\mathbf{4}} \mathbf{I N N}$, was followed using in situ small-angle $\mathrm{X}$-ray scattering during heating. The samples were placed into capillaries and placed into an X-ray transparent furnace. The samples were then heated at a heating rate of less than $2{ }^{\circ} \mathrm{C}$ per minute stepwise, and at each temperature step $\left(5{ }^{\circ} \mathrm{C}\right.$ intervals) a pattern was taken for $900 \mathrm{~s}$.

The SAXS results for $\mathbf{C l}$ INN were already reported in previous studies. ${ }^{19,20,34}$ The size of the silica nanoparticles is obtained from the scattering intensity towards low values of the scattering vector $q$. Second, at higher $q$-values (at approximately $q=15 \mathrm{~nm}^{-1}$ ), a broad peak is visible from which the typical distance between the silica tetrahedrons can be observed. Between these two regimes, at about $q=6 \mathrm{~nm}^{-1}$, a short range order peak is present which is attributed to the typical inter-particle distance of the imidazolium ligands due to $\pi-\pi$ stacking (Fig. 6b). ${ }^{19,20}$ Since the arrangement of the imidazolium ligands is of primary interest in our investigation, Fig. $6 \mathrm{~b}$ only shows the scattering intensities in the $q$-range between 2 and $15 \mathrm{~nm}^{-1}$. In good agreement with our previous observation that Cl_INN is stable in air up to $290{ }^{\circ} \mathrm{C},{ }^{15,16}$ we observe in Fig. $6 \mathrm{~b}$ no change in the structure when heating under vacuum from room temperature to $200{ }^{\circ} \mathrm{C}$. Neither the intensity nor the position of the short range order peak at about $q=6 \mathrm{~nm}^{-1}$ exhibits any change, and the structure at the nanometer scale is maintained. Equally, no degradation of the material was observed at the macroscopic scale.

Differently, the SAXS data of the second reference compound, (BuMeIm) $)_{2} \cdot \mathbf{C u C l}_{4}$, are strongly dependent on temperature (Fig. 6a): all crystalline peaks disappeared at temperatures higher than $150{ }^{\circ} \mathrm{C}$, in good agreement with DSC results. It should be noted that at temperatures above $100{ }^{\circ} \mathrm{C}$, an additional ordered phase seems to develop, visible by one additional peak at about $q=8.3 \mathrm{~nm}^{-1}$ (arrow in Fig. 6a). Whereas the peaks from the original crystalline phase vanished between 140 and $150{ }^{\circ} \mathrm{C}$, this additional phase seems to be slightly more stable: the peak is still visible at $150{ }^{\circ} \mathrm{C}$ and disappears in the temperature range between 150 and $160{ }^{\circ} \mathrm{C}$.

The temperature dependence of the SAXS intensities of the thermochromic material, $\mathbf{C u C l}_{\mathbf{4}} \mathbf{I N N}$, is shown in Fig. 6c. Here, a linear scale of the intensities is chosen for better visibility. For the material at room temperature, one small short range order peak is visible at about $q=10 \mathrm{~nm}^{-1}$, corresponding to a distance of the units in real space of about $d=0.6 \mathrm{~nm}$. Upon heating the sample to temperatures higher than $150{ }^{\circ} \mathrm{C}$, this peak disappears and a different peak evolves at about $q=$ $5.6 \mathrm{~nm}^{-1}$, corresponding to a distance of the units in real space of about $d=1.1 \mathrm{~nm}$. There is no gradual shift of the peak position, which indicates a phase transition from one structure to another. This clearly suggests a configurational change of the copper(II) chloride complexes from a square planar configuration to a compressed tetrahedral configuration, as known for thermochromic copper(II) chloride compounds. ${ }^{35}$ 

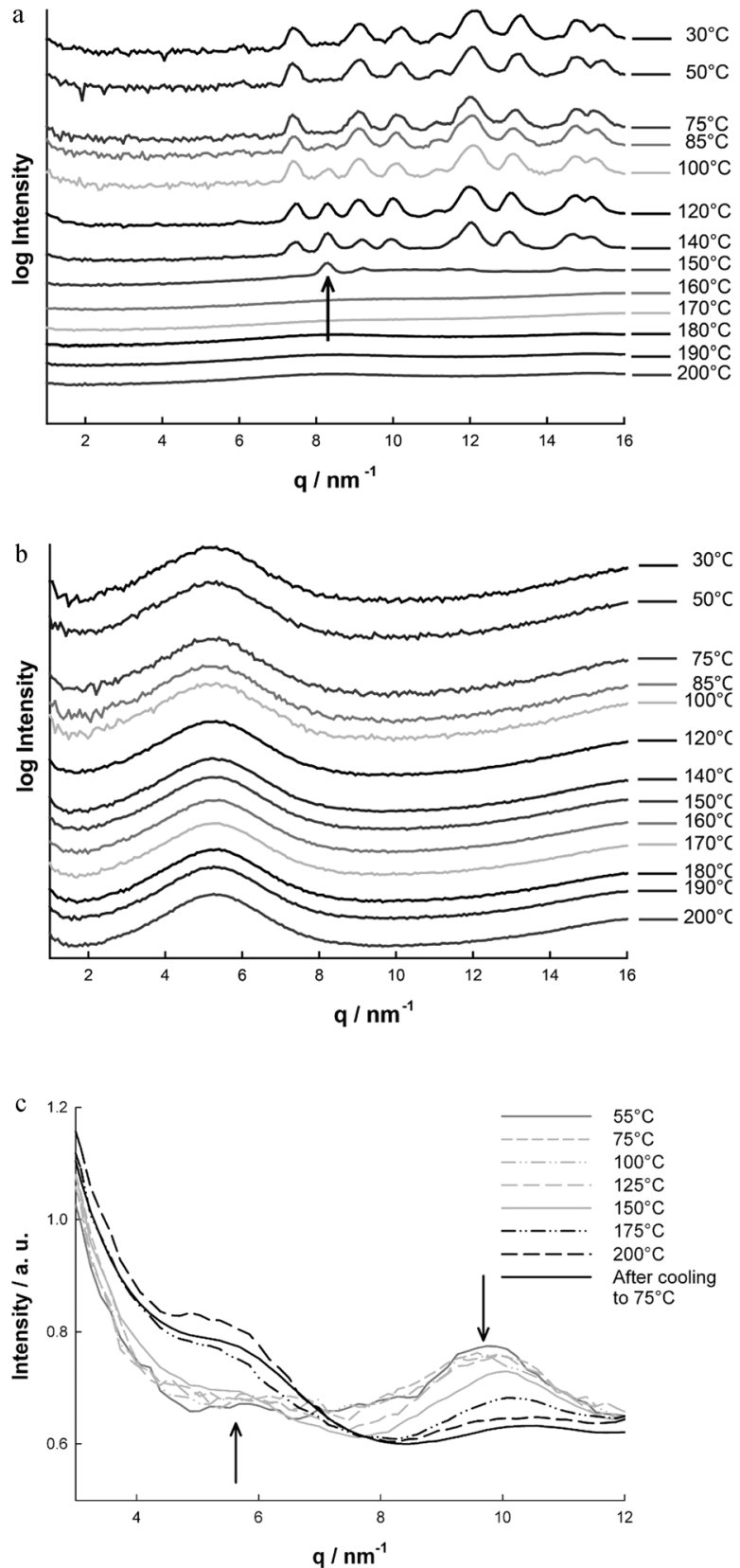

Fig. 6 SAXS intensities depending on temperature (measured in situ): (a) (BuMeIm) $)_{2} \cdot \mathrm{CuCl}_{4}$, the arrow indicates a peak from an additional phase, (b) Cl_INN shows no structural change, and (c) $\mathrm{CuCl}_{4}$ INN , the arrows indicate a phase transition from planar (small distance, peak at $10 \mathrm{~nm}^{-1}$ ) to tetragonal configuration (large distance, peak at $5 \mathrm{~nm}^{-1}$ ). The curves in (a) and (b) are shifted for better visibility.

Thus, the change observed in SAXS would characterize the evolution from a plane arrangement (with a lower distance) to the more space demanding tetragonal arrangement at higher temperatures. This transformation is completed at temperatures higher than $175^{\circ} \mathrm{C}$, which is in perfect agreement with the appearance of thermochromic behavior, i.e. the color change from green to yellow. In contrast to the reference compound, thermochromic material $\mathbf{C u C l}_{\mathbf{4}} \mathbf{I N N}$ is stable up to a temperature of $200{ }^{\circ} \mathrm{C}$. This suggests that the confined space between nanoparticles does not only allow thermochromic behavior at significantly higher temperatures than in pure diethylammonium copper tetrachloride, but also stabilizes the material itself.

The introduction of a complexing agent, $\mathrm{CuCl}_{2} \cdot 2 \mathrm{H}_{2} \mathrm{O}$, into the Cl_INN material is inducing two effects on the short-range order within the material. On the one hand, the intensity of short-range order is weaker in the thermochromic material $\mathbf{C u C l}_{4} \mathbf{I N N}$ than in the reference material $\mathbf{C l}$ INNN. On the other hand, but only at low temperature, the position of the short range order peak is located at higher $q$-values in the thermochromic material $\mathbf{C u C l}_{\mathbf{4}} \mathbf{I N N}$ than in the reference material Cl_INN, located at $10 \mathrm{~nm}^{-1}$ and $6 \mathrm{~nm}^{-1}$, respectively; while at temperatures higher than $150{ }^{\circ} \mathrm{C}$, the peak of the short range order for both the reference material and the thermochromic material is located at around $6 \mathrm{~nm}^{-1}$.

The complexation of the chloride anions induces most probably a re-organization, and thus a de-organization, of the imidazolium counter-parts as compared to the material without copper. This change and weakening of the short-range order in the material were verified by a strong change in the photoluminescence properties of the material. Indeed before complexation of the copper, the Cl_INN material shows a strong emission band at $400 \mathrm{~nm}$, for an excitation at $360 \mathrm{~nm}$ induced by the $\pi-\pi$ stacking of the imidazolium units in the inter-particle space (see ESI $\dagger$ ). The quantum yield associated with this emission of Cl_INN was estimated to be around $26 \% .{ }^{19}$ In contrast, a very weak emission can hardly be detected for $\mathbf{C u C l}_{4 \_} \mathbf{I N N}$ (see ESI $\dagger$ ). This emission is associated with a quantum yield near to zero $(0.5 \%)$.

We assume that, the imidazolium moieties are involved in the coordination sphere of the square planar copper at room and low temperatures, in a similar way to the solvent molecules for reported thermochromic ammonium copper tetrachloride..$^{35}$ This interaction could explain that the electrons of the aromatic rings do not interact with each other anymore as they are involved in the coordination of the copper. As a consequence of this lack of $\pi-\pi$ stacking, no luminescence can be observed for $\mathbf{C u C l}_{\mathbf{4}} \mathbf{I N N}$.

After the configuration change, from a square planar to a tetrahedral copper configuration, the imidazolium aromatic rings are no longer involved in the coordination sphere of the copper. However, large tetrahedral copper complexes are still present between the imidazolium rings, which could explain that the short-range order after heating of the material remains much weaker as in the Cl_INN material.

\section{Conclusions}

Imidazolium Nanoparticle Networks containing chloride anions (Cl_INN) were used for the complexation of copper dichloride. The complexation reaction resulted in an intense green color for the material, characteristic of the presence of square-planar copper tetrachloride complexes. With increasing temperature, a color change from green to yellow was observed at around $180{ }^{\circ} \mathrm{C}$. The color change indicates a configuration change of the copper tetrachloride complex, from green square-planar to a more 
space-demanding yellow tetrahedral arrangement. This thermochromic behavior is irreversible. This resulted in an increase of the distance of the ligands, which is visible by a sudden shift of the short-range order peaks in the SAXS patterns.

The silica nanoparticles are acting as substrates and are inducing the organization of the material. They are crucial for the thermochromic behavior as well as for the stability of the material.

The reference material without nanoparticles, bis-(butylmethylimidazolium)copper(II) tetrachloride, decomposes at $166{ }^{\circ} \mathrm{C}$ under air and thus no thermochromic behavior could be observed.

The thermochromic material is stable under air up to $250{ }^{\circ} \mathrm{C}$ and can also be easily processed into thin films. This is of particular interest for further technical applications. Therefore, this study is intended to open the door for the development of INN materials as optical sensors.

\section{Acknowledgements}

JA and HP thank the Austrian science funds FWF (project number I449) for financial support.

\section{References}

1 M. R. Jones and C. A. Mirkin, Nature, 2012, 491, 42-43.

2 S. Liu and Z. Tang, J. Mater. Chem., 2010, 20, 24-35.

3 M. P. Pileni, J. Phys. Chem. B, 2001, 105, 3358-3371.

4 L. Wang, L. Xu, H. Kuang, C. Xu and N. A. Kotov, Acc. Chem. Res., 2012, 45, 1916-1926.

5 M. R. Jones, K. D. Osberg, R. J. MacFarlane, M. R. Langille and C. A. Mirkin, Chem. Rev., 2011, 111, 3736-3827.

6 M.-P. Pileni, Nat. Mater., 2003, 2, 145-150.

7 W. Feng, L.-D. Sun and C.-H. Yan, Langmuir, 2011, 27, 3343-3347.

8 S. I. Lim and C.-J. Zhong, Acc. Chem. Res., 2009, 42, 798-808.

9 D. Zhang and R. Pelton, Langmuir, 2012, 28, 3112-3119.

10 R. Abargues, S. Albert, J. L. Valdes, K. Abderrafi and J. P. Martinez-Pastor, J. Mater. Chem., 2012, 22, 22204-22211.

11 K. M. Schulz, S. Abb, R. Fernandes, M. Abb, A. G. Kanaras and O. L. Muskens, Langmuir, 2012, 28, 8874-8880.

12 B. P. Pichon, P. Louet, O. Felix, M. Drillon, S. Begin-Colin and G. Decher, Chem. Mater., 2011, 23, 3668-3675.

13 M.-R. Gao, S.-R. Zhang, J. Jiang, Y.-R. Zheng, D.-Q. Tao and S.-H. Yu, J. Mater. Chem., 2011, 21, 16888-16892.
14 S. Guo and S. Sun, J. Am. Chem. Soc., 2012, 134, 2492-2495.

15 M. Litschauer and M.-A. Neouze, Monatsh. Chem., 2008, 139, 1151-1156.

16 M. Litschauer and M.-A. Neouze, J. Mater. Chem., 2008, 18, 640-646.

17 M.-A. Neouze, J. Mater. Chem., 2010, 20, 9593-9607.

18 J. Roeser, M. Kronstein, M. Litschauer, A. Thomas and M.-A. Neouze, Eur. J. Inorg. Chem., 2012, 5305-5311.

19 M. Czakler, M. Litschauer, K. Foettinger, H. Peterlik and M.-A. Neouze, J. Phys. Chem. C, 2010, 114, 21342-21347.

20 M. Litschauer, M. Puchberger, H. Peterlik and M.-A. Neouze, J. Mater. Chem., 2010, 20, 1269-1276.

21 B. Feichtenschlager, S. Pabisch, H. Peterlik and G. Kickelbick, Langmuir, 2012, 28, 741-750.

22 S. Pabisch, B. Feichtenschlager, G. Kickelbick and H. Peterlik, Chem. Phys. Lett., 2012, 521, 91-97.

23 P. Zare, A. Stojanovic, F. Herbst, J. Akbarzadeh, H. Peterlik and W. H. Binder, Macromolecules, 2012, 45, 2074-2084.

24 X. Li, Y. Zhou, Z. Zheng, X. Yue, Z. Dai, S. Liu and Z. Tang, Langmuir, 2009, 25, 6580-6586.

25 R. Ouyang, S. A. Bragg, J. Q. Chambers and Z.-L. Xue, Anal. Chim. Acta, 2012, 722, 1-7.

26 S. Gadzuric, M. Vranes and S. Dozic, Sol. Energy Mater. Sol. Cells, 2012, 105, 309-316.

27 X. Wei, L. Yu, D. Wang, X. Jin and G. Z. Chen, Green Chem., 2008, 10, 296-305.

28 J. H. Day, Chem. Rev., 1968, 68, 649-657.

29 G. F. Starkulla, S. Klenk, M. Butschies, S. Tussetschlaeger and S. Laschat, J. Mater. Chem., 2012, 22, 21987-21997.

30 C. M. Tollan, R. Marcilla, J. A. Pomposo, J. Rodriguez, J. Aizpurua, J. Molina and D. Mecerreyes, ACS Appl. Mater. Interfaces, 2009, 1, 348-352.

31 R. Bhattacharya, M. Sinha Ray, R. Dey, L. Righi, G. Bocelli and A. Ghosh, Polyhedron, 2002, 21, 2561-2565.

32 T. Sasaki, C. Zhong, M. Tada and Y. Iwasawa, Chem. Commun., 2005, 2506-2508.

33 H. Wang, B. Wang, C.-L. Liu and W.-S. Dong, Microporous Mesoporous Mater., 2010, 134, 51-57.

34 M. Litschauer, H. Peterlik and M.-A. Neouze, J. Phys. Chem. C, 2009, 113, 6547-6552.

35 R. D. Willett, J. A. Haugen, J. Lebsack and J. Morrey, Inorg. Chem., 1974, 13, 2510-2513. 